\title{
Association between osteoarthritis of the hand and knee in a population of skeletons from London
}

\author{
H A Waldron
}

\begin{abstract}
Objective-To examine the relation between osteoarthritis of the hand and knee in a group of skeletons dating to the 18th and early 19th centuries from two sites in London.

Methods-This was a case-control study of 115 cases and controls matched for age and sex. Cases were skeletons with osteoarthritis of the hands; cases and controls were assessed for the presence of osteoarthritis of any compartment of the knee.

Results-Cases had a significant odds ratio (OR) for osteoarthritis of the knee compared with controls: OR 5.98, 95\% confidence interval (CI) 1.25 to 56.37 . Cases with osteoarthritis of the distal interphalangeal (DIP), proximal interphalangeal (PIP), and first carpometacarpal (CMC1) joints had increased but nonsignificant odds ratios for osteoarthritis of the knee in the order DIP > PIP > CMC1. Cases with multifocal osteoarthritis of the hands had a significantly increased odds ratio for osteoarthritis of the knee: OR $11.67,95 \%$ CI 1.13 to 379.57 .

Conclusions-The study confirms the association between osteoarthritis of the hand and the knee seen in contemporary populations and suggests that it is not of very recent origin.
\end{abstract}

(Ann Rheum Dis 1997;56:116-118)

A subset of osteoarthritis in which the hand and knee are both involved has been recognised clinically for many years and was confirmed epidemiologically by Cushnagen and Dieppe ${ }^{1}$ for a group of women, but not men, attending a rheumatology clinic. The association was studied more recently by Hirsch and her colleagues in the participants of the Baltimore longitudinal study of aging. ${ }^{2}$ These investigators examined joints of the hand radiologically and identified three groups: those with osteoarthritis of the distal interphalangeal joint (DIP), proximal interphalangeal joint (PIP), and first carpometacarpal joint (CMC1) and they also defined a fourth group of individuals with at least two of these joints affected. They found significant crude odds ratios for knee osteoarthritis for all four groups, the highest value being for the group with multiple joint involvement.

Osteoarthritis is a disease which occurs commonly in the skeleton and is straightforward to diagnose; on these grounds it has been a disease to which palaeopathologists have given a good deal of attention. It occurs at all periods but some changes in the pattern of osteoarthritis seem to have taken place over time. For example, osteoarthritis of the hip was more common than osteoarthritis of the knee in the medieval period, but in post-medieval skeletons the converse is the case. ${ }^{34}$ Osteoarthritis of the knee is the more common form in contemporary populations but whether the change from the earlier pattern is due to a increase in osteoarthritis of the knee or to a decrease in osteoarthritis of the hip is at present unclear from the skeletal evidence.

To see whether the association between osteoarthritis of the hand and the knee was of recent origin, it has been studied in a group of skeletons recovered from archaeological sites dating from the late 18th and early 19 th centuries.

\section{Methods}

The skeletons came from two sites in London, Farringdon Street and Christ Church, Spitalfields. The cemetery in Farringdon Street was an overflow cemetery for the St Bride's Parish and was used from the middle of the 18 th century to the early 19 th century; the crypt at Spitalfields was used for burials between 1729 and 1857 .

The skeletons had all been sexed and aged, using standard anthropological methods ${ }^{5}$ for other purposes before this study was undertaken; osteoarthritis was never used as an aging criterion. The skeletons were placed into one of two 10 year age bands (35-44 or 45-54) or an age group of $55+$. Osteoarthritis was diagnosed whenever a joint surface showed the presence of eburnation since this is considered to be pathognomonic of the condition in palaeopathological material.

Cases were taken as skeletons with osteoarthritis of the hands and for which age and sex had been reliably ascertained. It was a condition of entry to the study that both hands and both knee joints were present, and that at least one surface representative of each of the
Accepted for publication 7 November 1996 
joints of the hands (including the carpal bones) was also present.

One hundred and fifteen cases fulfilled the entry criteria: 48 males and 67 females. Forty one of the cases were from Farringdon Street and 74 from Spitalfields. For each case, a control which definitely did not have osteoarthritis of the hands and which was matched for sex and age was selected from among the remaining skeletons at the appropriate site. It was, of course, necessary that the knee joints were present in each control skeleton.

The number of cases and controls with osteoarthritis of the knee was then determined with the author blind as to their case/control status. The result was positive whichever of the compartments of the knee was affected-the patellofemoral (PF) or either of the tibiofemoral (TF) compartments. Following this, crude odds ratios were calculated for cases with osteoarthritis of the hand (any joint) having osteoarthritis of the knee. In addition, the data were analysed maintaining pair matching using McNemar's test. In order to obtain data similar to those of Hirsch et al, odds ratios were calculated for subgroups of cases having osteoarthritis of the DIP, PIP, and CMC1 joints.

The effect of having different numbers of joints affected was also studied. For each case the total number of joints with osteoarthritis was counted, the number referring to the number of anatomical sites involved. Thus a skeleton having osteoarthritis of, say, three different DIPs, CMC1, and the first metacarpophalangeal joint (MCP1) was considered to have three joints (not five) affected. Bilateral disease was counted only once.

In order to investigate the possibility of a bias due to age differences between the cases and controls, the prevalence of another highly age related condition-diffuse idiopathic skeletal hyperostosis (DISH) - was determined in both groups using diagnostic criteria described elsewhere. ${ }^{5}$

All statistical tests were carried using either EPI-6 or KwikStat Professional 4.12 for Windows.

\section{Results}

Of the cases, 11 (four male and seven female) had osteoarthritis of the knee compared with only two controls (both female). In eight of the cases and one of the controls, the TF joint was involved. For the cases as a whole the odds ratio was 5.98 , exact $95 \%$ confidence intervals (CI) being 1.25 to 56.37 (see table). When stratified by sex, the Mantel-Haenszel weighted odds ratio was 6.02 (95\% CI 1.21 to 40.30). The matched pairs analysis gave a $\chi^{2}$ value (with Yates' correction) of 4.92 ( $\mathrm{P}=$ $0.027)$. When considering only those skeletons with $\mathrm{TF}$ disease, the odds ratio was 10.36 , exact $95 \%$ CI 1.20 to 480.76 ; the matched pairs $\chi^{2}$ value (with Yates' correction) was 4.00 , $\mathrm{P}=0.046$.

Forty seven of the cases had osteoarthritis of the DIPs, 24 had osteoarthritis of the PIPs, and 58 had osteoarthritis of CMC1. The numbers of these which also had osteoarthritis of the knee were 7,4 , and 7 , respectively. The odds
Crude odds ratio (OR) and exact $95 \%$ confidence intervals (CI) for the association of hand and knee osteoarthritis

\begin{tabular}{lc}
\hline Group & OR $(95 \%$ CI $)$ \\
\hline All hand and knee & $5.98(1.25$ to 56.37$)$ \\
DIP and knee & $2.80(0.66$ to 13.77$)$ \\
PIP and knee & $2.40(0.46$ to 10.48$)$ \\
CMC1 and knee & $1.82(0.43$ to 8.95$)$ \\
Two or more joints and knee & $11.67(1.13$ to 379.57$)$
\end{tabular}

DIP/PIP, distal/proximal interphalangeal joint; CMC1, 1st carpometacarpal joint.

ratios were found to decrease such that DIP > PIP > CMC1; the 95\% CI in all cases, however, included unity, indicating that they were not statistically significant at the conventional level (table). The majority of cases had multifocal disease, 66 having osteoarthritis of at least two joints of the hands.

DISH was present in eight of the cases (seven from Spitalfields) and in seven of the controls (six from Spitalfields), a difference which is obviously not significant.

\section{Discussion}

The association between osteoarthritis of the hand and the knee, which has been demonstrated in the contemporary population, ${ }^{12}$ has been confirmed in this study of a group of skeletons of individuals who lived in London during the 18th and early 19th centuries. This pattern of osteoarthritis, then, cannot be said to be of very recent origin. It is certain that the socioeconomic conditions and occupations of the individuals represented in this sample of skeletons and those of the patients studied by Hirsch and her colleagues were very different, and they may also have differed between my two sample subsets, since the Spitalfields group is known to have included a large number of Huguenots who were engaged in the weaving industry to a considerable extent, while the Farringdon Street population was much more heterogeneous. The proportion of cases and controls with osteoarthritis of the knee was not significantly different at either site, however: $10.8 \%$ and $1.2 \%$, respectively at Spitalfields, and $7.4 \%$ and $2.4 \%$ at Farringdon Street. Taken together, these data suggest that social factors and occupation may not be major determinants of the common expression of osteoarthritis in the hand and knee.

Osteoarthritis of the hand and the knee are both related to obesity ${ }^{7-9}$ and in their study Hirsch and her colleagues ${ }^{2}$ adjusted their data for body mass index. There is no prospect of being able to do this in a group of skeletons, and the influence of this confounder cannot be estimated. The other important potential confounders in this study are those of age and sex. While it is easy to control for both in modern epidemiology, the palaeoepidemiologist faces something of a problem. Sexing of skeletons in which the pelvis and/or the skull are present should not generally cause an experienced osteologist any difficulties, but it is well known that the aging of skeletons can be very difficult. The aging was carried out by the same observer and there is no a priori reason to suppose that any systematic bias was introduced by the presence of osteoarthritis of the hands; 
indeed, it had been carried out before the present study was undertaken. The presence of osteoarthritis was never used as a criterion for aging, although this is sometimes done by other anthropologists; since our interest has been in the prevalence and pattern of osteoarthritis in past populations, using the presence of this disease to assess the age of an individual would negate much of our epidemiological work. The fact that there was no difference in the prevalence of DISH between the two groups, however, supports the contention that the differences found in the prevalence of osteoarthritis of the knee are not due to age acting as a confounder, although of course it cannot be considered absolutely conclusive. There is no reason to suppose that the use of DISH as a surrogate for age introduced any additional confounding as this condition is not related to osteoarthritis.

The results of this study emphasise the likelihood that individuals with multifocal disease also have osteoarthritis of the knee, confirming the data of Hirsch et al. ${ }^{2}$ And although the odds ratios for DIP, PIP, and CMC1 were not significant at the $5 \%$ level, it is at least of interest that the rank order tallied exactly with that of Hirsch and her colleagues, and the greater propensity for those with osteoarthritis of the DIPs to have knee osteoarthritis confirms what is known clinically about generalised osteoarthritis. In some of the cases not all the DIPs or PIPs were present-although at least eight DIPs or six PIPs were always represented by one or other of the reciprocal joint surfaces-so it is possible that the prevalence of osteoarthritis at these joints may have been underestimated and if this were indeed the case, then the odds ratios would also be underestimates.

In another paper, Hochberg and his colleagues ${ }^{10}$ have shown that osteoarthritis of the hands is related to osteoarthritis of the hips. This was not the case here; in a supplementary analysis, eight of the cases were found also to have osteoarthritis of the hip, compared with seven of the controls. The reasons for this discrepancy are not at all clear, and it would be helpful to examine this relation in another sample.

1 Cushnagen J, Dieppe P. Study of 500 patients with limb joint osteoarthritis. I. Analysis by age, sex, and distribution

2 Hirsch R, Lethbridge-Cejku M, Scott WW, Reichle R, Plato

$\mathrm{CC}$, Tobin J, et al. Association of hand and knee osteoarthritis: evidence for a polyarticular disease subset. Ann Rheum Dis 1996;55:25-9.

3 Rogers J, Dieppe P. Is tibiofemoral osteoarthritis in the knee joint a new disease? Ann Rheum Dis 1994;53:612-3

4 Waldron T. Changes in the distribution of osteoarthritis over historical time. Int J Osteoarcheol 1995;5:385-9.

5 Workshop of European Anthropologists. Recommendations for age and sex diagnosis of skeletons. J Hum Evol 1980;9:517-49.

6 Rogers J, Waldron T. A field guide to joint diseases in archaeology. Chichester: John Wiley \& Sons, 1995.

7 Felson, DT. Epidemiology of hip and knee osteoarthritis. Epidemiol Rev 1988;10:1-28.

8 Anderson JT, Felson DT. Factors associated with osteoarthritis of the knee in the first national health and nutrition examination survey (HANES I). Evidence for an association with overweight, race and physical demands of association with overweight, race and phy
work. Am J Epidemiol 1994;139:119-29.

9 Hochberg MC, Lethbridge-Ceku M, Plato CC, Wigley FM, Tobin JD. Factors associated with osteoarthritis of the hand in males:data from the Baltimore longitudinal study of aging. Am J Epidemiol 1991;134:1121-7.

10 Hochberg MC, Lane NE, Pressman AR, Genant HK, Scott JC, Nevitt MC. The association of radiographic changes of osteoarthritis of the hand and hip in elderly women. J Rheumatol 1995;22:2291-4. 